\title{
BMJ Open Reoperation after oesophageal cancer surgery in relation to long-term survival: a population-based cohort study
}

\author{
Maartje van der Schaaf, ${ }^{1}$ Maryam Derogar, ${ }^{1}$ Asif Johar, ${ }^{1}$ Martin Rutegård, ${ }^{1}$ \\ James Gossage, ${ }^{2}$ Robert Mason, ${ }^{2}$ Pernilla Lagergren, ${ }^{1}$ Jesper Lagergren ${ }^{1,2,3}$
}

To cite: van der Schaaf $M$, Derogar M, Johar A, et al. Reoperation after oesophageal cancer surgery in relation to long-term survival: a population-based cohort study. BMJ Open 2014;4: e004648. doi:10.1136/ bmjopen-2013-004648

- Prepublication history for this paper is available online. To view these files please visit the journal online (http://dx.doi.org/10.1136/ bmjopen-2013-004648)

Received 9 December 2013 Revised 14 February 2014 Accepted 26 February 2014

CrossMark

\begin{abstract}
${ }^{1}$ Department of Molecular Medicine and Surgery, Karolinska Institutet, Stockholm, Sweden

${ }^{2}$ Department of Surgery, St Thomas' Hospital, London, UK

${ }^{3}$ Division of Cancer Studies, King's College London, London, UK
\end{abstract}

Correspondence to Dr Maartje van der Schaaf; maartje.van.der.schaaf@ki.se

\section{ABSTRACT}

Objectives: The influence of reoperation on long-term prognosis is unknown. In this large population-based cohort study, it was aimed to investigate the influence of a reoperation within 30 days of oesophageal cancer resection on survival even after excluding the initial postoperative period.

Design: This was a nationwide population-based retrospective cohort study.

Setting: All hospitals performing oesophageal cancer resections during the study period (1987-2010) in Sweden.

Participants: Patients operated for oesophageal cancer with curative intent in 1987-2010.

Primary and secondary outcomes: Adjusted HRs of all cause, early and late mortality up to 5 years after reoperation following oesophageal cancer resection.

Results: Among 1822 included patients, the 200 (11\%) who were reoperated had a $27 \%$ increased HR of all-cause mortality (adjusted $\mathrm{HR} 1.27,95 \% \mathrm{Cl} 1.05$ to 1.53 ) and $28 \%$ increased HR of disease-specific mortality (adjusted HR $1.28,95 \% \mathrm{Cl} 1.04$ to 1.59 ), compared to those not reoperated. Reoperation for anastomotic insufficiency in particular was followed by an increased mortality (adjusted $\mathrm{HR} 1.82,95 \% \mathrm{Cl} 1.19$ to 2.76 ).

Conclusions: This large and population-based nationwide cohort study shows that reoperation within 30 days after primary oesophageal resection was associated with increased mortality, even after excluding the initial 3 months after surgery. This finding stresses the need to consider any actions that might prevent complications and reoperation after oesophageal cancer resection.

\section{INTRODUCTION}

Despite recent developments in multimodal therapy, surgical tumour resection is still the mainstay of treatment for localised oesophageal cancer. ${ }^{1}$ While postoperative mortality has decreased to less than $5 \%$ in recent years, ${ }^{2}$ oesophageal resection still carries a considerable risk for postoperative complications, some of which require reoperation. ${ }^{134}$

\section{Strengths and limitations of this study}

- The study had a population-based cohort design.

- It was possible to adjust for several confounding factors through comprehensive data collection from medical records and registries.

- Data on complications other than reoperation were missing.

- The retrospective design was a limitation.

Major postoperative complications are known to negatively influence short-term survival, ${ }^{2}$ but evidence of the influence of such complications on long-term survival is inconsistent. ${ }^{5-8}$ A recent study from our group suggested that surgical complications after oesophageal cancer resection might be an independent predictor for a poorer longterm survival. ${ }^{9}$ Any potential effect of reoperation in lowering long-term survival after oesophagectomy could be mediated by several biological mechanisms, for example, the additional surgical trauma could further trigger an inflammatory response that could lower the efficacy of bodily defence mechanisms, including destruction and removal of circulating tumour cells, and thus pave the way for early recurrence, ${ }^{10}$ and the major surgical complications that cause the need for reoperation might directly facilitate tumour recurrence, for example, anastomotic insufficiency might entail direct tumour spread. ${ }^{911}$ Therefore, we hypothesised that reoperation within 30 days after initial oesophageal resection negatively influences long-term survival.

\section{METHODS}

Study design

This was a retrospective population-based cohort study. The study cohort has previously been presented in detail. ${ }^{12}{ }^{13}$ All patients having undergone oesophagectomy for 
oesophageal cancer during the period 1987-2010 in Sweden were included in the study. Eligible patients were followed up until death or end of the study (28 February 2012), whichever occurred first.

\section{Study population}

Patients with oesophageal cancer were identified from the Swedish Cancer Registry, a registry with $98 \%$ nationwide coverage of patients with oesophageal cancer. ${ }^{14} 15$ Tumours of the gastric cardia were not included. Oesophageal cancer was defined by the diagnosis code $150.0,150.8$ and 150.9 in the seventh version of the International Classification of Diseases (ICD7). The identified patients were linked with the Swedish Patient Registry to include only those who underwent oesophageal resection in the final study cohort. Our group has recently reported that the Patient Registry has a positive predictive value of $99.6 \%$ for assessing oesophageal cancer resection. ${ }^{16}$ Detailed information on tumour characteristics and surgical details was acquired through manual scrutiny of medical records from the operation charts and histopathology reports, with accompanying referral notes, retrieved from all relevant hospitals throughout Sweden. ${ }^{12} 13$ One reviewer, who was kept blinded for the study outcome to ensure objectivity, reviewed all histopathological reports according to a predefined protocol to ensure uniformity. The accuracy of the histopathological review was assessed by two researchers who independently reviewed 100 patient records, showing high accuracy ( $>90 \%$ concordance) ${ }^{13}$ The Patient Registry was used to obtain information on reoperations after the primary oesophageal resection and on comorbidities. Detailed information about indications for reoperation was not available. To calculate survival time after oesophagectomy, dates of death were collected from the Swedish Causes of Death Registry. This register is complete and is updated continuously, facilitating the availability of accurate dates of death. The unique 10-digit Swedish personal identity number, assigned to every resident in Sweden since $1947,{ }^{17}$ was used for linkage of individuals between registries and for identification of the patients' hospital records.

\section{Study exposure and outcome}

The exposure was defined as any open or minimally invasive reoperation within 30 days of initial oesophageal cancer resection. Exposure was defined according to the Classification of Surgical Procedures. More specifically, reoperation was categorised as: (1) explorative laparotomy (ICD10 JAH00, JAK00), (2) explorative thoracotomy (ICD10 GAB13, GAB96, GAB10), (3) reoperation for bleeding (ICD10 JWE00, GWE00), (4) reoperation for anastomotic insufficiency (ICD10 JWF00, GWF00, DWF00, (5) reoperation for wound revision (ICD10 JWA00) or (6) reoperation for deep infection (ICD10 GWC00, GCW01, JWC00; table 1).

The study outcomes were all-cause early-specific, latespecific and disease-specific mortality. 'Early
Table 1 Categorisation of the 248 reoperations within 30 days after initial surgery in a cohort of 1822 patients undergoing oesophagectomy between 1987 and 2010 in Sweden, with follow-up until 28 February 2012

\begin{tabular}{lc}
\hline Type of reoperation & Number (\%) \\
\hline Total number of reoperations & $248(100)$ \\
Explorative laparotomy & $47(19)$ \\
Explorative thoracotomy & $11(4)$ \\
Reoperation for bleeding & $22(9)$ \\
Reoperation for anastomotic insufficiency & $43(17)$ \\
Laparotomy & 3 \\
Thoracotomy & 1 \\
Unknown/other & 39 \\
Reoperation for infection & $8(3)$ \\
Reoperation for wound revision & $50(20)$ \\
Wound revision for bleeding & 15 \\
Wound revision for infection & 5 \\
Wound dehiscence & 7 \\
Unknown & 23 \\
Other reoperations & $75(30)$ \\
\hline
\end{tabular}

postoperative mortality' was defined as any death occurring within 90 days of initial surgery, while 'late mortality' was defined as any death between 90 days and 5 years of the primary resection. 'Disease specific mortality' was defined as death of tumour recurrence occurring between 90 days and 5 years of surgery. If a cause of death included oesophageal cancer (diagnosis codes 150 according to ICD7) in the Swedish Causes of Death Registry, we assumed that patients died of tumour recurrence. We also analysed the impact of each of the most common types of reoperations on mortality between 90 days and 5 years of surgery in subgroup analyses. Since tumour recurrence is a less likely explanation for mortality 5 years and later after oesophageal cancer surgery, we decided to use 5 years as a cut-off.

\section{Statistical analysis}

Survival was calculated using the Kaplan-Meier method, and differences in survival between the survival curves of patients with and without reoperation were evaluated using the log-rank test. In a Cox proportional hazards regression model, HRs with $95 \%$ CIs were calculated, including adjustment for potential confounding factors in a multivariable model. In the Cox model, the proportionality assumption was tested. The factors adjusted for were nine known prognostic factors. They were categorised as follows: (1) age (categorised into three groups: $<65,65-75$ or $>75$ years); (2) sex; (3) comorbidity (including any of the following: hypertension, ischaemic heart disease, cardiac failure, chronic obstructive pulmonary disease, asthma, diabetes, former cancer diagnosis, HIV, liver disease and renal disease; and categorised into three groups: none, one, or two or more); (4) tumour stage (classified according to the sixth version of the Union for International Cancer Control-TNM (tumour, node, metastasis) classification 
and categorised into four groups: 0-I, II, III or IV); (5) histological type of tumour (categorised into two groups: squamous cell carcinoma or adenocarcinoma); (6) neoadjuvant therapy (yes or no) data on the type of neoadjuvant therapy used, that is, chemoradiotherapy or chemotherapy, was not available, but in Sweden, the use of chemoradiotherapy has dominated whenever neoadjuvant therapy has been used; (7) surgical radicality (R0 or not R0); (8) surgeon volume ( $<9$ or $\geq 9$ per year) To avoid selecting a suitable cut-off for surgeon annual volume, we simply chose to use the median as the cut-off and (9) calendar period (1987-1996 or 19962005). We also considered lymph node harvest as a potential confounder, but this variable did not significantly influence the results ( $\chi^{2} \mathrm{p}$ value 0.687 ), and since there was a substantial rate of missing data on lymph node harvest, we decided not to include this variable in the final multivariable model. Information on comorbidities was obtained from the Swedish Patient Register, information on tumour stage and histological type of tumour, surgical radicality and neoadjuvant treatment was extracted from histopathological records and accompanying referral notes. ${ }^{16}$ Missing values in the covariates were treated as a separate group in the Cox regression model. ${ }^{18}$ A sensitivity analysis was performed to compare the impact of categorising missing as a separate group against removing these missing values from the analysis.

All statistical analyses were performed using STATA V.11 for Mac (STATAcorp College Station, Texas, USA).

\section{RESULTS}

Patients

Some 2195 patients were identified as eligible in the study cohort of patients with oesophageal cancer who underwent resection in Sweden during the study period. After exclusion of 373 patients (17\%) for whom medical records were not available or exposure data were missing, $1822(83 \%)$ patients remained for final analysis. Of these, 200 patients $(11 \%)$ were exposed for reoperation (in total 248 reoperations) within 30 days of the primary oesophageal resection (table 1 ).

There were no major differences between the groups with and without reoperation regarding the distribution of sex, age, comorbidity, tumour stage, tumour histology, neoadjuvant therapy, hospital volume or calendar period (table 2). Among the 1484 patients who died during the entire study period, $1246(84 \%)$ had documented tumour recurrence, which means that the all-cause mortality within 5 years closely mirrors disease-specific mortality. There were no missing values for reoperation (exposure) and missing values in covariates were missing at random. A sensitivity analysis was performed to compare the impact of categorising missing as a separate group against removing these missing values from the analysis, and the results were similar (data not shown). In the Cox model, the proportionality assumption was tested and the model satisfied the assumption.
Reoperation and risk of mortality

Among the 208 patients (11\%) who died within 90 days of surgery, 54 (26\%) underwent reoperation. Reoperation was a risk factor for 90-day mortality after adjustment for confounding factors (HR 3.05, 95\% CI 2.22 to 4.17$)$. Among the $1276(79 \%)$ patients who died between 90 days and 5 years after surgery, $117(10 \%)$ were reoperated. Among the 122 who died after 5 years of surgery, $5(4 \%)$ were reoperated. The log-rank test comparing the Kaplan-Meier survival curves of patients with and without reoperation between 90 days and 5 years after surgery revealed a statistically significantly increased mortality in the reoperated group $(p<0.0001$; figure 1 and table 3 ).

As presented in table 3 , there was a $27 \%$ increased hazard of mortality during the period 90 days to 5 years after surgery after adjustment for all nine potential confounding factors (crude HR 1.22, adjusted HR 1.27, $95 \%$ CI 1.05 to 1.53 ; table 3 ). During the follow-up period, $954(74 \%)$ patients died of reported tumour recurrence. The disease-specific mortality within 90 days and 5 years of surgery was increased by $28 \%$ among patients who were reoperated (adjusted HR 1.28, 95\% CI 1.04 to 1.59 ; table 3 ). The proportional hazard assumption, tested using a non-zero slope, and time varying covariates were satisfied, and there were no statistically significant interaction effects with reoperation (data not shown).

\section{Reoperation and risk of mortality-subgroup analyses of most common reoperations}

In a subgroup analysis of the three most common types of reoperations, that is, exploratory laparotomy, reoperation for anastomotic insufficiency and wound revision, the point HRs were increased for each type of reoperation (table 4), and patients reoperated for anastomotic insufficiency in particular had a statistically significantly increased hazard of mortality (adjusted HR 1.82, 95\% CI 1.19 to 2.76 ).

\section{DISCUSSION}

This is, to the best of our knowledge, the first study addressing reoperation in relation to late mortality after primary oesophageal cancer resection, and it revealed an increased long-term all-cause and disease-specific mortality in patients who underwent reoperation compared with those who did not. Patients who underwent reoperation due to anastomotic insufficiency experienced a particularly high hazard of mortality.

Among the strengths of this study is the populationbased design in which most patients who underwent oesophageal cancer surgery in Sweden during 19872010 were included. The follow-up for mortality was complete by virtue of the availability of personal identity numbers for Swedish residents, together with the fully complete Swedish Causes of Death Registry. Another major strength is the possibility to adjust for several 
Table 2 Characteristics of 1822 patients undergoing oesophagectomy between 1987 and 2010 in Sweden, with follow-up until 28 February 2012

\begin{tabular}{|c|c|c|c|}
\hline \multirow[b]{2}{*}{ Characteristics } & \multicolumn{2}{|l|}{ Number (\%) } & \multirow[b]{3}{*}{$p$ Value $^{\star}$} \\
\hline & No reoperation & Reoperation & \\
\hline Total & $1622(89)$ & $200(11)$ & \\
\hline \multicolumn{4}{|l|}{ Sex } \\
\hline Men & $1211(75)$ & $151(75)$ & \multirow[t]{2}{*}{0.8} \\
\hline Women & 411 (25) & 49 (25) & \\
\hline \multicolumn{4}{|l|}{ Age } \\
\hline$\leq 64$ & $754(46)$ & $93(47)$ & \multirow[t]{3}{*}{0.9} \\
\hline $65-75$ & $615(38)$ & 78 (39) & \\
\hline$>75$ & $253(16)$ & $29(14)$ & \\
\hline \multicolumn{4}{|l|}{ Comorbidity $†$} \\
\hline None & $832(51)$ & $107(54)$ & \multirow[t]{3}{*}{0.8} \\
\hline 1 & $542(34)$ & $63(31)$ & \\
\hline$\geq 2$ & $248(15)$ & $30(15)$ & \\
\hline \multicolumn{4}{|l|}{ Stage } \\
\hline $0-1$ & $339(20)$ & $41(20)$ & \multirow[t]{5}{*}{0.9} \\
\hline II & $532(33)$ & 71 (35) & \\
\hline III & $399(25)$ & $46(23)$ & \\
\hline IV & $127(8)$ & $13(7)$ & \\
\hline Missing§ & $225(14)$ & $29(15)$ & \\
\hline \multicolumn{4}{|l|}{ Histology } \\
\hline Adenocarcinoma & $645(40)$ & $70(35)$ & \multirow[t]{3}{*}{0.09} \\
\hline Squamous cell carcinoma & $880(54)$ & $123(62)$ & \\
\hline Missing§ & $97(6)$ & $7(3)$ & \\
\hline \multicolumn{4}{|l|}{ Neoadjuvant therapy } \\
\hline None & $677(42)$ & $85(43)$ & \multirow[t]{4}{*}{0.4} \\
\hline Radiotherapy & $154(9)$ & $26(13)$ & \\
\hline Chemoradiotherapy & 302 (19) & $35(17)$ & \\
\hline Missing§ & $489(30)$ & $54(27)$ & \\
\hline \multicolumn{4}{|l|}{ Radicality } \\
\hline Ro & 1135 (69) & $137(68)$ & \multirow[t]{3}{*}{0.7} \\
\hline Not Ro & 251 (16) & $30(15)$ & \\
\hline Missing§ & $236(15)$ & $33(17)$ & \\
\hline \multicolumn{4}{|l|}{ Surgeon volume } \\
\hline$<9$ per year & $875(54)$ & $122(61)$ & \multirow[t]{2}{*}{0.06} \\
\hline$\geq 9$ per year & 747 (46) & 78 (39) & \\
\hline \multicolumn{4}{|l|}{ Calendar period } \\
\hline 1987-1990 & $234(14)$ & $34(17)$ & \multirow[t]{5}{*}{0.2} \\
\hline 1991-1994 & 302 (19) & $43(22)$ & \\
\hline 1995-1999 & $330(20)$ & $49(25)$ & \\
\hline 2000-2005 & $382(24)$ & 37 (19) & \\
\hline $2006-2010$ & $374(23)$ & 37 (19) & \\
\hline
\end{tabular}

${ }^{*} \chi^{2}$ of the difference between groups.

†Comorbidity included: hypertension, ischaemic heart disease, cardiac failure, chronic obstructive pulmonary disease, asthma, diabetes, former cancer diagnosis, HIV, liver disease and renal disease.

¥Categorised according to the sixth version of the Union for International Cancer Control (UICC)-TNM (tumour, node, metastasis) classification.

§Missing values of covariates were missing at random and considered as a separate group.

known prognostic factors, which reduces the risk of confounding. Moreover, the exposure and outcome were predefined, which reduces the risk of chance findings and decreases the risk of systematic errors owing to misclassification. Some limitations of the study require a discussion. The retrospective clinical data collection imposes a risk of misclassification and selection bias. The researchers involved in gathering the clinical information had, however, no link with the participating hospitals and were not involved in the patient care, which decreases these risks. A risk of residual confounding by known prognostic factors or confounding by unknown factors cannot be excluded in observational research due to the lack of randomisation. There was, for example, no access to information on preoperative performance status and nutritional status, which might have influenced the results. ${ }^{19}$ Another limitation was the lack of information on complications, and thus the 


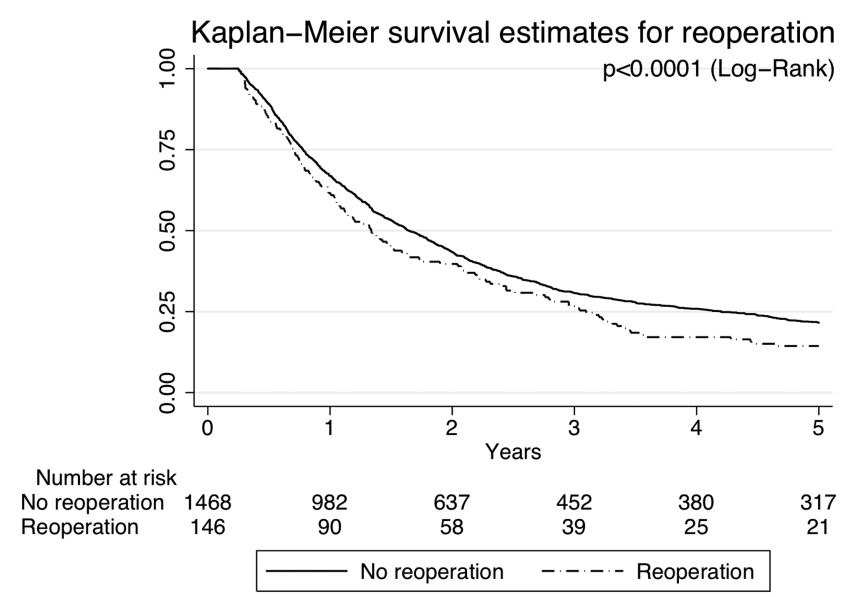

Figure 1 Kaplan-Meier survival curve for survival between 90 days and 5 years with respect to occurrence of reoperation among 1614 patients undergoing oesophageal cancer surgery in 1987-2010 in Sweden.

indication for the reoperations. Although the long study period offered good statistical power, it also entailed a risk of bias by changes in surgical techniques and standards in patients' care over time. To counteract such effects, we adjusted all HRs for calendar period. The limited rate of exposure to reoperation still meant that the exposure could not be further subcategorised because of power issues. Finally, the use of a cut-off of 30 days of surgery for assessing reoperation might result in missing later reoperations. However, we decided before the study was initiated to use a cut-off that was likely to be directly associated with the oesophagectomy, but was yet not too short. Since there is no agreed on cut-off for capturing early reoperations associated with surgery, we instead use a commonly used cut-off for short-term mortality, which is traditionally 30 days.

The finding of the prognostic role of reoperations after excluding the initial postoperative period is a novel finding that should encourage further research. It stresses the need for preventive measures to reduce the need
Table 4 HRs with $95 \%$ Cls of mortality between 90 days and 5 years in a subgroup analyses of the most common types of reoperations after oesophagectomy, based on 1822 patients undergoing oesophageal cancer surgery in 1987-2012 in Sweden

\begin{tabular}{lll}
\hline Type of reoperation & $\begin{array}{l}\text { Number of } \\
\text { patients }(\%)\end{array}$ & HR $(95 \% \mathbf{C l})^{\star}, \boldsymbol{\dagger}$ \\
\hline $\begin{array}{l}\text { Exploratory laparotomy } \\
\text { Reoperation for }\end{array}$ & $47(19)$ & $1.17(0.82$ to 1.67$)$ \\
$\begin{array}{l}\text { anastomotic } \\
\text { insufficiency }\end{array}$ & $1.82(1.19$ to 2.76$)$ \\
Wound revision & $50(20)$ & $1.32(0.87$ to 2.00$)$ \\
\hline
\end{tabular}

*Adjusted for sex, age, comorbidities, tumour stage, histology, neoadjuvant therapy, radicality, surgeon volume and calendar period.

†Missing values of covariates were missing at random and considered as a separate group.

for reoperation. In this population, the three most commonly performed types of reoperation were explorative laparotomy (19\%), reoperation for anastomotic leak $(17 \%)$ and wound revision $(20 \%)$. The results of the subgroup analyses showed that especially patients undergoing reoperation for anastomotic insufficiency had an increased risk of mortality. There is some evidence that anastomotic insufficiency entails direct tumour spread and seeding of remaining viable tumour cells in patients with colon cancer. ${ }^{11} 20$ This might explain the higher mortality in patients with reoperation for anastomotic insufficiency.

Several studies have shown that a higher surgeon or hospital volume reduces postoperative mortality and morbidity. ${ }^{12}$ 21-24 High volume surgery is facilitated by centralisation of the care for patients with oesophageal cancer. Centralisation might be an effective measure for prevention of severe postoperative complications. A recent study showed that patients with comorbidity that compromises the cardiovascular status leading to a compromised perfusion of organs (eg, hypertension, diabetes, congestive heart failure and renal failure) have a higher risk of

Table 3 HR with $95 \%$ Cls of mortality after oesophagectomy with respect to occurrence of reoperation, based on 1822 patients undergoing oesophageal cancer surgery in 1987-2012 in Sweden

\begin{tabular}{|c|c|c|c|}
\hline Reoperation & Number of patients (\%) & Number of events (\%)‡ & HR $(95 \% \mathrm{Cl})^{*}$ \\
\hline \multicolumn{4}{|l|}{ All stages } \\
\hline$<90$ days & $1822(100)$ & 208 (11) & \\
\hline Crude & & & 3.17 (2.32 to 4.32$)$ \\
\hline Multivariable*,$\dagger$ & & & 3.05 (2.22 to 4.17$)$ \\
\hline$\geq 90$ days -5 years & $1614(89)$ & $1276(79)$ & \\
\hline Crude & & & 1.22 (1.02 to 1.47$)$ \\
\hline Multivariable,$\dagger$ & & & 1.27 (1.05 to 1.53$)$ \\
\hline$\geq 90$ days -5 years disease specific & $1292(71)$ & $954(74)$ & \\
\hline Crude & & & 1.26 (1.03 to 1.57$)$ \\
\hline Multivariable ${ }^{\star}, \dagger$ & & & 1.28 (1.04 to 1.59$)$ \\
\hline
\end{tabular}

${ }^{*}$ Adjusted for sex, age, comorbidities, tumour stage, histology, neoadjuvant therapy, radicality, surgeon volume and calendar period. †Missing values of covariates were missing at random and considered as a separate group. ‡Event means death. 
anastomotic leak. This finding indicates that preoperative optimisation of the cardiovascular status might also decrease the risk of severe complications requiring reoperation. ${ }^{25}$ No previous studies have, to the best of our knowledge, addressed the influence of reoperations on long-term survival in patients with oesophageal cancer. However, the main indication for reoperation is the occurrence of severe postoperative complications, and a few previous studies have assessed the role of such complications on long-term survival. These have provided conflicting results; some studies have reported a worse long-term prognosis after surgical complications, ${ }^{79}$ medical complications, ${ }^{10}$ or concurrent surgical and medical complication, ${ }^{4}$ while others have not found any such effect. ${ }^{6} 8$ These differences might be due to the differences in classification of the severity of the complications and missing information on interventions.

One biological mechanism that might explain the decreased long-term survival after reoperation is that the additional surgical injury reduces the protection against seeding of tumour cells, including activation of natural killer cells and other anticarcinogenic factors. ${ }^{26}$ Furthermore, it is possible that additional surgery triggers an elevated inflammatory response that might in turn stimulate the growth of microtumours and induce tumour recurrence and death from recurrence. ${ }^{10}$ Another potential mechanism considers certain complications. Finally, blood transfusion has been linked with a worse long-term mortality and increased cancer recurrence in different types of cancer. ${ }^{27-30}$ Unfortunately, we did not have information on blood transfusion in this study, but it can be assumed that patients returning to theatre are more likely to receive blood transfusion, and speculatively, blood transfusion may be a mechanism that contributes to the main finding of this study.

In conclusion, this nationwide and population-based cohort study with adjustment for several potential confounding factors indicates that reoperation is associated with an increased hazard of mortality even after the initial 3 months of the oesophageal cancer resection. This finding warrants more research, but further stresses the need to consider any actions that might prevent complications requiring reoperation after the primary surgery in patients with oesophageal cancer.

Contributors MvdS and JL had full access to all the data in the study and take responsibility for the integrity of the data and the accuracy of the data analysis. MvdS, JL, PL, MR and MD were involved in the study concept and design. PL, JL, MvdS and MD contributed to the acquisition of data. MvdS, $\mathrm{JL}$ and $\mathrm{AJ}$ contributed to the analysis and interpretation of data. MvdS and $\mathrm{JL}$ were involved in the drafting of the manuscript. JG, RMM, MR and PL were involved in the critical revision of the manuscript for important intellectual content. AJ and MD conducted statistical analysis. JL and PL obtained funding.

Funding This study was funded by the Swedish Cancer Society (grant number 521-2012-3241) and the Swedish Research Council (grant number 12-0748).

Competing interests None.

Ethics approval The Regional Ethical Review Board in Stockholm, Sweden approved the study.
Provenance and peer review Not commissioned; externally peer reviewed.

Data sharing statement Statistical codes and the dataset are available from the primary investigator JL (jesper.lagergren@ki.se) at the Upper GI Research Group, Department of Molecular Medicine and Surgery, Karolinska Institutet, Stockholm Sweden, who will provide a permanent and citable home for the dataset.

Open Access This is an Open Access article distributed in accordance with the Creative Commons Attribution Non Commercial (CC BY-NC 3.0) license, which permits others to distribute, remix, adapt, build upon this work noncommercially, and license their derivative works on different terms, provided the original work is properly cited and the use is non-commercial. See: http:// creativecommons.org/licenses/by-nc/3.0/

\section{REFERENCES}

1. Wu PC, Posner MC. The role of surgery in the management of oesophageal cancer. Lancet Oncol 2003;4:481-8.

2. Schieman C, Wigle DA, Deschamps C, et al. Patterns of operative mortality following esophagectomy. Dis Esophagus 2012;25:645-51.

3. Gebski V, Burmeister B, Smithers BM, et al. Survival benefits from neoadjuvant chemoradiotherapy or chemotherapy in oesophageal carcinoma: a meta-analysis. Lancet Oncol 2007;8:226-34.

4. Lerut T, Moons J, Coosemans W, et al. Postoperative complications after transthoracic esophagectomy for cancer of the esophagus and gastroesophageal junction are correlated with early cancer recurrence: role of systematic grading of complications using the modified Clavien classification. Ann Surg 2009;250:798-807.

5. Hirai T, Yamashita Y, Mukaida $\mathrm{H}$, et al. Poor prognosis in esophageal cancer patients with postoperative complications. Surg Today 1998;28:576-9.

6. Ferri LE, Law S, Wong $\mathrm{KH}$, et al. The influence of technical complications on postoperative outcome and survival after esophagectomy. Ann Surg Oncol 2006;13:557-64.

7. Rizk NP, Bach PB, Schrag D, et al. The impact of complications on outcomes after resection for esophageal and gastroesophageal junction carcinoma. J Am Coll Surg 2004;198:42-50.

8. Ancona E, Cagol M, Epifani M, et al. Surgical complications do not affect longterm survival after esophagectomy for carcinoma of the thoracic esophagus and cardia. J Am Coll Surg 2006;203:661-9.

9. Rutegard M, Lagergren P, Rouvelas I, et al. Intrathoracic anastomotic leakage and mortality after esophageal cancer resection: a population-based study. Ann Surg Oncol 2012;19:99-103.

10. Lagarde SM, de Boer JD, ten Kate FJW, et al. Postoperative complications after esophagectomy for adenocarcinoma of the esophagus are related to timing of death due to recurrence. Ann Surg 2008;247:71-6.

11. Walker KG, Bell SW, Rickard MJ, et al. Anastomotic leakage is predictive of diminished survival after potentially curative resection for colorectal cancer. Ann Surg 2004;240:255-9.

12. Derogar M, Sadr-Azodi O, Johar A, et al. Hospital and surgeon volume in relation to survival after esophageal cancer surgery in a population-based study. J Clin Oncol 2013;31:551-7.

13. Rouvelas I, Zeng W, Lindblad M, et al. Survival after surgery for oesophageal cancer: a population-based study. Lancet Oncol 2005;6:864-70.

14. Barlow L, Westergren K, Holmberg L, et al. The completeness of the Swedish Cancer Register: a sample survey for year 1998. Acta Oncol 2009;48:27-33.

15. Lindblad M, Ye W, Lindgren A, et al. Disparities in the classification of esophageal and cardia adenocarcinomas and their influence on reported incidence rates. Ann Surg 2006;243:479-85.

16. Lagergren K, Derogar M. Validation of oesophageal cancer surgery data in the Swedish Patient Registry. Acta Oncol 2012;51:65-8.

17. Ludvigsson JF, Otterblad-Olausson P, Pettersson BU, et al. The Swedish personal identity number: possibilities and pitfalls in healthcare and medical research. Eur J Epidemiol 2009;24:659-67.

18. Cohen JC, Cohen P. Applied multiple regression/correlation analysis for the behavioral sciences. 2nd edn. Hillsdale, NJ: Lawrence Erlbaum, 1983.

19. Grotenhuis BA, Wijnhoven BP, Grune F, et al. Preoperative risk assessment and prevention of complications in patients with esophageal cancer. J Surg Oncol 2010;101:270-8.

20. McArdle CS, McMillan DC, Hole DJ. Impact of anastomotic leakage on long-term survival of patients undergoing curative resection for colorectal cancer. Br J Surg 2005;92:1150-4. 
21. Birkmeyer JD, Siewers AE, Finlayson EV, et al. Hospital volume and surgical mortality in the United States. $N$ Engl J Med 2002;346:1128-37.

22. Birkmeyer JD, Stukel TA, Siewers AE, et al. Surgeon volume and operative mortality in the United States. N Engl J Med 2003;349:2117-27.

23. Brusselaers N, Mattsson F, Lagergren J. Hospital and surgeon volume in relation to long-term survival after oesophagectomy: systematic review and meta-analysis. Gut 2013. Published Online First. doi:10.1136/gutjnl-2013-306074

24. Rouvelas I, Lagergren J. The impact of volume on outcomes after oesophageal cancer surgery. ANZ J Surg 2010;80:634-41.

25. Kassis ES, Kosinski AS, Ross $P$ Jr, et al. Predictors of anastomotic leak after esophagectomy: an analysis of the society of thoracic surgeons general thoracic database. Ann Thorac Surg 2013;96:1919-26.
26. Abramovitch R, Marikovsky M, Meir G, et al. Stimulation of tumour growth by wound-derived growth factors. Br J Cancer 1999;79:1392-8.

27. Tachibana $\mathrm{M}$, Tabara $\mathrm{H}$, Kotoh $\mathrm{T}$, et al. Prognostic significance of perioperative blood transfusions in resectable thoracic esophageal cancer. Am J Gastroenterol 1999;94:757-65.

28. Liu L, Wang Z, Jiang S, et al. Perioperative allogenenic blood transfusion is associated with worse clinical outcomes for hepatocellular carcinoma: a meta-analysis. PLoS ONE 2013;8:e64261.

29. Uccella S, Ghezzi F, Cromi A, et al. Perioperative allogenic blood transfusions and the risk of endometrial cancer recurrence. Arch Gynecol Obstet 2013;287:1009-16.

30. Acheson AG, Brookes MJ, Spahn DR. Effects of allogeneic red blood cell transfusions on clinical outcomes in patients undergoing colorectal cancer surgery: a systematic review and meta-analysis. Ann Surg 2012;256:235-44. 